\title{
Rückfallrate metamphetaminabhängiger PatientInnen nach niedrigschwelligem Drogenentzug - Ergebnisse einer mittelfristigen Katamnese
}

\section{Silke Datzer \\ Roland Härtel-Petri \\ Martin Schiller \\ Manfred Wolfersdorf}

\author{
Relapses of Metamphetamine Dependents After Inpatient Low Treshold \\ Withdrawal
}

\section{Zusammenfassung}

Im Beitrag wird eine an 57 Metamphetaminabhängigen durchgeführte Untersuchung zur Frage der Rückfallhäufigkeiten nach niedrigschwelligem Drogenentzug vorgestellt. Mittels eines Fragebogens wurden im Verlauf (vor und nach Entgiftungsbehandlung, Katamnese nach durchschnittlich 6,3 Monaten) spezifische Daten erfasst. Das Drogenkonsumverhalten zeigte in wichtigen Parametern eine deutliche Verbesserung.

\section{Schliüsselwörter}

Metamphetaminabhängigkeit · Niedrigschwelliger Drogenentzug · Rückfallrate

\section{Abstract}

57 inpatient metamphetamine dependents were surveyed by a different questionnaire three times (admission for detoxication, discharge after detoxication, half year after release on average). The relapses after a low treshold withdrawal were evaluated by specific items. Important parameters of drug-dependence have shown an improvement.

Key words

Metamphetamine Dependence · Withdrawal · Relapse

\section{Einleitung}

In den letzten Jahren ist eine zunehmende Rolle der Amphetamine auf dem Drogenmarkt zu verzeichnen. Für Deutschland belegen dies z.B. die Zahlen des Bundeskriminalamtes Wiesbaden [1]. Der Anteil der Amphetamine unter den polizeilich erstauffälligen KonsumentInnen harter Drogen ist seit 1992 stetig gestiegen und liegt seit 1996 nach den Opiaten an zweiter Stelle. Die sichergestellten Drogenmengen haben sich für Amphetamine zwischen 1992 und 1998 verdreifacht, für Heroin aber im gleichen Zeitraum halbiert. Auch der Drogenbericht der Deutschen Referenzstelle für die Europäische Beobachtungsstelle für Drogen und Drogensucht [2] berichtet über eine Zunahme der Amphetaminabhängigen.

Amphetamine verfügen kurzfristig gesehen über Wirkungen, die der heutigen Fun- und Leistungsgesellschaft sehr entgegen kommen: Sie reduzieren den Appetit und das Schlafbedürfnis, stär- ken das Selbstvertrauen und erhöhen die allgemeine Leistungsfähigkeit [3]. Zudem sind sie billig und einfach herzustellen. Dies hat sicherlich zu ihrer starken Verbreitung beigetragen. An ungünstigen Wirkungen ist das besonders häufige Auftreten psychotischer Störungen hervorzuheben [4,5]. Nach Klee [6] kommt es bei zwei Dritteln der Personen mit regelmäßigem Konsum innerhalb von drei Jahren zu einer so genannten Amphetaminpsychose.

Nach einer Welle des besonders durch die Medien bekannt gewordenen Amphetaminderivates „Ecstasy“ [7] ist nun in den letzten Jahren ein Anstieg für das Metamphetamin zu verzeichnen [3,6,8-12]. Metamphetamin ist das Derivat unter den Amphetaminen, das über den stärksten Stimulanzcharakter und im Vergleich zu Ecstasy weniger halluzinogene Wirkungen verfügt. 
Bisher liegen über das Problem der Amphetaminabhängigkeit wenige Untersuchungen vor und wenn, dann hauptsächlich auf Ecstasy bezogen. Um der Entwicklung auf dem Drogenmarkt im Rahmen der Drogenpolitik gerecht werden zu können, sind daher dringend Untersuchungen über diese sich neu entwickelnde Klientel notwendig.

\section{Material und Methoden}

Die nachfolgend dargestellten Ergebnisse basieren auf einer Untersuchung, die auf der niedrigschwelligen Drogenentzugsstation des Bezirkskrankenhauses Bayreuth (BKH) durchgeführt wurde. Im Zeitraum September 1999 bis März 2000 wurden alle PatientInnen, die von der Station mit der Diagnose einer Metamphetaminabhängigkeit entlassen wurden, in die Studie aufgenommen. Die Diagnosestellung erfolgte nach ICD-10 (F 15), als Hauptdroge musste Metamphetamin eindeutig im Vordergrund stehen. Zudem konnten 18 Metamphetaminabhängige aus einer kurz zuvor auf der Station durchgeführten Studie mit einbezogen werden. Die Stichprobe umfasste insgesamt $n=57$ Probanden, die zu drei Zeitpunkten mittels eines spezifischen Fragebogens untersucht wurden. Die Fragebogen wurden von den Probanden zum größten Teil selbständig ausgefüllt, vereinzelt wurden Items durch die Einschätzung des Arztes erfasst (z.B. körperlicher Allgemeinzustand). Die drei Zeitpunkte waren 1. Aufnahme zur Entgiftungsbehandlung, 2. Entlassung nach der Entgiftungsbehandlung, 3. in einem durchschnittlichen Katamnesezeitraum von 6,3 Monaten (range: 3,5-13,5 Monate) nach der Entlassung. Zur Nachbefragung wurden 45 ProbandInnen $(78,9 \%)$ der Ausgangsstichprobe erreicht. Erfasst wurden soziodemographische, somatische, suchtbezogene und forensische Daten, deren Veränderungen im Verlauf verglichen wurden. Die Auswertung wurde mittels SPSS durchgeführt. Ein Vergleich der Responder $(n=45)$ mit den nicht antwortenden ehemaligen Patienten hinsichtlich soziodemographischer Daten zeigte keine Unterschiede.

\section{Ergebnisse}

\section{Soziodemographische Daten}

In der Ausgangsstichprobe $(n=57)$ waren 70,2\% Männer und $29,8 \%$ Frauen. Das Durchschnittsalter lag insgesamt bei 23,5 Jahren (Männer 24 Jahre, Frauen 22,3 Jahre), das durchschnittliche Drogeneinstiegsalter betrug 17,9 Jahre (Männer 17,3 Jahre, Frauen 18,1 Jahre).

Der Zeitpunkt der Nachbefragung lag zwischen 3,5 und 13,5 Monaten, im Mittel bei sechs Monaten und drei Wochen. Die Daten der Nachbefragungsgruppe $(n=45)$ bez. Geschlechterverteilung, Durchschnittsalter, Drogeneinstiegsalter und Behandlungsdauer unterschieden sich dabei nicht signifikant von der Ausgangsstichprobe $(n=57)($ s. Tab. 1$)$.

\section{Dauer der Abstinenz nach Entlassung}

Bei der Nachbefragung bezeichneten sich $42,2 \%$ als abstinent. $13,3 \%$ hatten innerhalb der ersten Woche nach der Entlassung den Drogenkonsum wieder begonnen. Innerhalb des ersten Monats wurden insgesamt $28,8 \%$ und in der Zeit bis zu sechs Monaten insgesamt $42,2 \%$ rückfällig. 4,4\% machten hierzu keine
Tab. 1 Soziodemographische Daten der Untersuchung

\begin{tabular}{lll}
\hline & $\begin{array}{l}\text { Ausgangsstichprobe } \\
(\mathbf{n = 5 7 )}\end{array}$ & $\begin{array}{l}\text { Responder } \\
(\mathbf{n = 4 5 )}\end{array}$ \\
\hline Männer/ & $70,2 \%$ & $71,1 \%$ \\
Frauen & $29,8 \%$ & $28,9 \%$ \\
\hline Durchschnittsalter & 23,5 Jahre & 23,6 Jahre \\
Männer/ & 24,0 Jahre & 24,6 Jahre \\
Frauen & 22,3 Jahre & 21,2 Jahre \\
\hline Erkrankungsdauer & 5,6 Jahre & 5,7 Jahre \\
Männer/ & 6,2 Jahre & 6,1 Jahre \\
Frauen & 4,2 Jahre & 4,7 Jahre \\
\hline
\end{tabular}

Angabe. Im Durchschnitt lag die Zeit des ersten Rückfalls bei 6,4 Wochen (s. Abb.1).

Bei der Entlassung waren 71,4\% drogenfrei und 28,6\% teilentgiftet. Innerhalb der ersten Woche nach der Entlassung waren 16\% der entgifteten und 7,1\% der teilentgifteten PatientInnen rückfällig geworden. Innerhalb der ersten vier Wochen waren dies 29\% der als drogenfrei und 14,3\% der als teilentgiftet Entlassenen.

\section{Drogenkonsum seit Entlassung}

33,3\% der Nachbefragten gaben an, seit der Entlassung keine Drogen mehr zu konsumieren, 15,5\% keine außer gelegentlich Cannabis. 17,7\% hatten zusätzlich gelegentlich auch andere Drogen konsumiert, aber deutlich weniger als vor der Entgiftungsbehandlung. Über regelmäßigen, aber ebenfalls deutlich reduzierten Drogenkonsum berichteten 2,2\%, keine Veränderung war bei $13,3 \%$ zu verzeichnen. Ein gesteigerter Konsum fand sich bei 6,6\%, eine zusätzliche Erweiterung des Drogenspektrums bei $2,2 \%$. Weitere 2,2\% machten keine Angabe.

$66,6 \%$ bezeichneten ihre Suchtschwere als deutlich reduziert, $26,6 \%$ als unverändert und $4,4 \%$ als verstärkt. Eine Person machte keine Angabe.

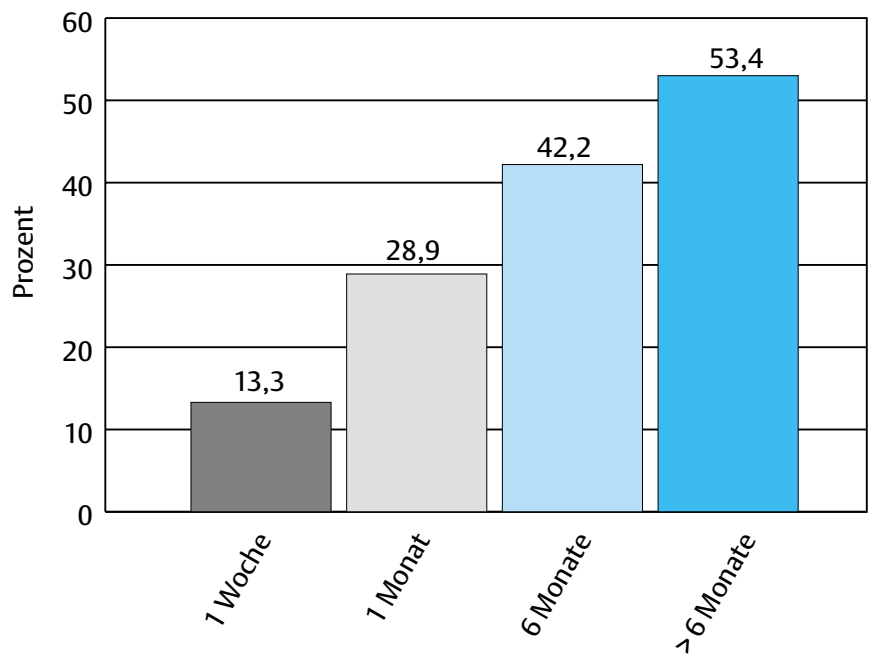

Abb. 1 Häufigkeitsverteilung der rückfällig gewordenen Patienten ( $n=24$ von $n=45$ Nachbefragten) zu verschiedenen Zeitpunkten nach der Entlassung. 
Vor der Behandlung auf der Drogenentzugsstation im Bezirkskrankenhaus Bayreuth haben $31 \%$ der Ausgangsstichprobe ( $\mathrm{n}=57$ ) Opiate zusätzlich konsumiert. Zum Zeitpunkt der Nachbefragung waren dies noch $16,7 \%(n=45)$. Der Anteil intravenös applizierender Metamphetaminabhängiger betrug zum Aufnahmezeitpunkt 52,4\%, bei der Nachbefragung nur noch $26,2 \%$ (s. Abb. 2).

\section{Neue Entzugsbehandlungen}

Eine erneute Entzugsbehandlung hatten insgesamt 33,3\% durchgeführt.

Neu aufgetretene Begleiterkrankungen: Bei 22,2\% der Nachbefragten waren neue Begleiterkrankungen aufgetreten (Psychosen, Herzrhythmusstörungen, wahnhafte Depression, Gewichtszunahme, Angstzustände, gastrointestinale Störungen, Alkoholismus). Zwei Probanden spezifizierten die aufgetretenen Erkrankungen nicht näher.

\section{Veränderungen seit der Entlassung in verschiedenen Lebensbereichen}

Es wurde nach Veränderungen (besser, gleich, schlechter) bezüglich des körperlichen Allgemeinzustandes, Suchtschwere, Partnerschaft, Berufs-, Ausbildungs- und Wohnsituation sowie juristischer und finanzieller Lage gefragt (s. Tab.2). Des Weiteren wurde speziell nach dem Nutzen (viel, wenig, gar nichts) der auf der Drogenentzugsstation im Bezirkskrankenhaus Bayreuth durchgeführten Entgiftungsbehandlung für einzelne Bereiche gefragt (s. Tab. 3).

\section{Suchtspezifische Hilfen seit der Entlassung}

Die Suchtberatung suchten 51,1\% auf, davon 78,2\% unregelmäßig und $21,7 \%$ regelmäßig. Eine ambulante Therapie machten $42,2 \%$, wovon $15,8 \%$ diese als erfolgreich beendet beurteilten. Eine stationäre Therapie machten $64,4 \%$ (da auf einige PatientInnen bei-

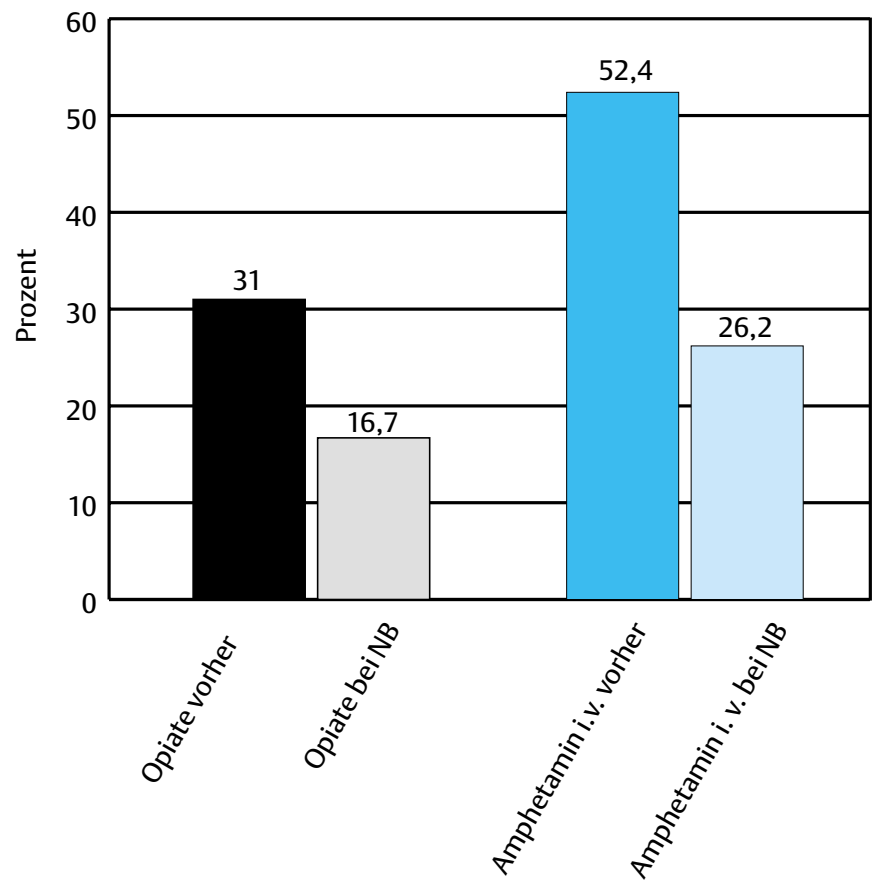

Abb. 2 Opiat-/Amphetaminkonsum (intravenös) vor der Entgiftung und bei der Nachbefragung (NB).
Tab. 2 Veränderungen seit der Entlassung in verschiedenen Lebensbereichen (Angaben in Prozent)

\begin{tabular}{|lcccc|}
\hline Bereich & besser & gleich & schlechter & keine Angabe \\
\hline körp. Allgemeinzustand & $\mathbf{5 7 , 7}$ & 28,8 & 11,3 & 2,2 \\
\hline Suchtschwere & $\mathbf{6 6 , 6}$ & 26,6 & 4,4 & 2,2 \\
\hline Partnerschaft & 31,0 & 51,1 & 11,1 & 6,6 \\
\hline berufl. Situation & 31,1 & 53,3 & 11,1 & 4,4 \\
\hline Ausbildung & 20,0 & 64,4 & 4,4 & 11,1 \\
\hline Wohnsituation & 17,7 & 73,3 & 4,4 & 2,2 \\
\hline Justiz & 31,0 & 51,1 & 11,1 & 6,6 \\
\hline Finanzen & $\mathbf{5 1 , 1}$ & 33,2 & 11,1 & 4,4 \\
\hline
\end{tabular}

Tab. 3 Nutzen der Entgiftung (Angaben in Prozent)

\begin{tabular}{lrrrr}
\hline Bereich & viel & wenig & nichts & keine Angabe \\
\hline allgemein & $\mathbf{7 3 , 3}$ & 4,4 & 20 & 2,2 \\
\hline Arbeit & 17,7 & 31,1 & 48,9 & 2,2 \\
\hline Partnerschaft & 22,2 & 35,5 & 31,1 & 11,1 \\
\hline Justiz & 35,5 & 28,8 & 26,6 & 8,8 \\
Gesundheit & $\mathbf{7 5 , 5}$ & 8,8 & 15,7 & 0 \\
\hline
\end{tabular}

des zutrifft, beträgt die Summe über 100\%), davon haben $11 \%$ diese erfolgreich abgeschlossen, 33\% sich noch in der Behandlung befunden und $54 \%$ diese nicht planmäßig beendet. Den Hausarzt suchten 11,1\% regelmäßig, 24,4\% unregelmäßig, 20\% nur anfangs und 35,5\% gar nicht auf. 8,8\% machten keine Angabe. $22,2 \%$ hatten unregelmäßig sonstige Hilfen aufgesucht (Reiki, Bachblütentherapie, meditative Gruppe) und 2 Personen diese regelmäßig.

\section{Diskussion}

Die Verteilung Männer/Frauen unterschied sich nicht wesentlich von anderen Studienergebnissen [13,14]. Das Durchschnittsalter der Untersuchungsgruppe war mit 23,5 Jahren etwa sechs bis acht Jahre deutlich jünger als in anderen Studien $[12,15,16]$. Hier spielen sicherlich verschiedene Settingparameter eine Rolle, je nachdem, ob es sich z.B. um eine stationär oder ambulant behandelte Klientel handelt. Doch könnte das sehr junge Durchschnittsalter auch als ein Charakteristikum dieser Subgruppe unter den Drogenabhängigen interpretiert werden. Die relativ häufig auftretenden psychotischen Störungen könnten zu einem schneller auftretenden und höheren Leidensdruck führen und dadurch das frühere Aufsuchen stationärer Einrichtungen bedingen.

Fast $43 \%$ der Nachbefragten bezeichneten sich noch als abstinent, was gegenüber anderen Untersuchungen [13] einen wesentlichen Unterschied darstellt. Ob dies im Zusammenhang mit dem Alter, drogenspezifischen Aspekten, konsumgruppenspezifischen Aspekten oder besserer Behandlungsansprechbarkeit steht, bleibt offen. Allerdings muss berücksichtigt werden, dass sich zum Nachbefragungszeitpunkt noch ein erheblicher Teil in stationären Einrichtungen befand. 
Kam es zu einem Suchtmittelrückfall, geschah dies in den meisten Fällen innerhalb der ersten vier Wochen nach der Entlassung. Die Rückfallquote der vollständig Entgifteten lag dabei höher als bei den Teilentgifteten. Ergebnisse zu einem längeren Verlauf wären hier sicherlich sinnvoll.

Neben der hohen Abstinenzquote ist es bei weiteren 20\% zu einer deutlichen Reduzierung des Drogenkonsums gekommen.

Der Anteil derer, die vor der Entgiftungsbehandlung Opiate missbrauchten, konnte auf die Hälfte reduziert werden, ebenso der Anteil derjenigen, die Amphetamine injizierten. Es konnte also insgesamt eine deutliche Verbesserung des Drogenkonsumverhaltens festgestellt werden. Katamnesen über einen längeren Zeitraum wären hier für die Zukunft wünschenswert.

Ein Drittel der PatientInnen hatte seit der Entlassung erneut eine Entzugsbehandlung durchgeführt, knapp die Hälfte von ihnen wieder auf der Station des BKH. Zum einen spricht die gezeigte Wiederkommensbereitschaft für das Konzept der Station, zum anderen konnte anscheinend mindestens wegbereitend die Motivation zur Aufnahme weiterer Behandlungsmöglichkeiten gefördert werden. Insgesamt gaben über drei Viertel der Nachbefragten auch an, sich gegebenenfalls erneut hier behandeln zu lassen.

Die Suchtberatungsstelle konsultierten bei der Nachbefragung weniger als vor der Behandlung. Dies ist vermutlich durch die Versorgung der PatientInnen durch weitere Einrichtungen zu erklären, spricht aber auch dafür, dass das bestehende ambulante Drogenhilfssystem diese Klientel nicht erreicht. Die Abbruchquote in stationären Therapieeinrichtungen ist mit fast ${ }^{2} / 3$ der PatientInnen sehr hoch. Obwohl das häufigere Antreten und Abbrechen von Behandlungen bei Drogenabhängigen typisch und ein Symptom ihrer Erkrankung ist, sollte dies auch als ein Hinweis gedeutet werden, dass ein dringender Bedarf einer Anpassung therapeutischer Konzepte besteht.

Bei fast $1 / 4$ war es in dem doch relativ kurzen Katamnesezeitraum zu neu aufgetretenen Begleiterkrankungen gekommen, dabei relativ häufig zu psychiatrischen Störungen (Psychosen, Depression, Angstzustände, Alkoholismus). Das heißt, dass auch bei den Metamphetaminabhängigen häufig psychiatrische Komorbidität besteht, was im ambulanten als auch stationären Drogenhilfsangebot Berücksichtigung finden muss und für die Notwendigkeit der klinischen Suchtmedizin integriert in die Psychiatrie spricht.

Weit über die Hälfte der Nachbefragten berichtete über eine Reduzierung ihrer Suchtschwere sowie eine Verbesserung des körperlichen Allgemeinzustandes. Auch die finanzielle Situation hatte sich bei gut $50 \%$ der PatientInnen verbessert. Etwa drei Viertel der Nachbefragten gaben an, dass ihnen die Behandlung auf der Station S 3 viel genutzt hätte.

Alle anderen Bereiche (Partnerschaft, berufliche Situation, Ausbildung, Wohnsituation) wurden überwiegend als gleichbleibend, aber auch nicht verschlechtert geschildert, wie bei fortlau- fender Drogenkarriere ja letztlich zu erwarten wäre. Doch auch letztgenannte Bereiche bieten wichtige Voraussetzungen für einen langfristig erfolgreichen Ausstieg aus dem Drogenmilieu.

Die Erarbeitung und Überprüfung weiterer therapeutischer Konzepte (z.B. eine spezielle Substitutionsbehandlung), die diese Aspekte verstärkt aufgreifen, wären für die Zukunft daher wünschenswert siehe auch [17]).

\section{Literatur}

${ }^{1}$ BKA-2001 Bundeskriminalamt Wiesbaden. Jahresberichte zur Rauschgiftkriminalität. http://www.BKA.de

${ }^{2}$ Deutsche Referenzstelle für die Europäische Beobachtungsstelle für Drogen und Drogensucht (DBDD). Bericht zur Drogensituation 2000. Institut für Therapieforschung (IFT) München, Deutsche Hauptstelle gegen die Suchtgefahren (DHS) Hamm, Bundeszentrale für gesundheitliche Aufklärung (BzgA) Köln 2000

${ }^{3}$ Hando J, Hall W. Patterns of amphetamine use in Australia. In: Klee $\mathrm{H}$ (Hrsg). Amphetamine Misuse. International Perspectives on Current Trends. Amsterdam: Overseas Publishers Association 1997

${ }^{4}$ Williamson S, Gossop M, Powis B, Griffiths P, Fountain J, Strang J. Adverse effects of stimulant drugs in a community sample of drug users. Drug and Alcohol Dependence 1997; 44: 87-94

${ }^{5}$ Wyatt SA, Ziedonis D. Psychological and Psychiatric Consequences of Amphetamines. In: Tarter RE, Ammerman RT (Hrsg). Handbook of substance abuse. Neurobehavioral Pharmacology. New York: Plenum Press 1998; 529-544

${ }^{6}$ Klee H. Patterns of amphetamine misuse in seven nations: Factors affecting growth and decline. In: Klee $\mathrm{H}$ (Hrsg). Amphetamine Misuse. International perspectives on current trends. Amsterdam: Overseas Publishers Association 1997; 291 - 320

${ }^{7}$ Thomasius R, Kraus D. Historische und epidemiologische Aspekte des Ecstasy-Konsums. In: Thomasius R (Hrsg). Ecstasy - Wirkungen, Risiken, Interventionen. Stuttgart: Enke 1999; 15-22

${ }^{8}$ Kraus L, Bauernfeind R. Konsumtrends illegaler Drogen in Deutschland: Daten aus Bevölkerungssurveys 1990-1995. Sucht 1998; 44: $169-182$

${ }^{9}$ Charnaud B, Griffith V. Levels of intravenous drug misuse among clients prescribed oral dexamphetamine or oral methadon: a comparison. Drug and Alcohol Dependence 1998; 52: 79-84

${ }^{10}$ Yoshida T. Use and misuse of amphetamines: an international overview. In: Klee $\mathrm{H}$ (Hrsg). Amphetamine Misuse. International perspectives on current trends. Amsterdam: Overseas Publishers Association 1997; 1 - 16

11 Darke S, Cohen J, Ross J, Hando J, Hall J. Transitions between routes of administration of regular amphetamine users. Addiction 1994; 89: $1077-1083$

12 Peters A, Davies T, Richardson A. Increasing popularity of injection as the route of administration of amphetamine in Edinburgh. Drug and Alcohol Dependence 1997; 48: 227-234

${ }^{13}$ Petri S. Niedrigschwelliger Drogenentzug. Ergebnisse einer 1-Jahreskatamnese bei 100 Patientinnen und Patienten einer Station für niedrigschwelligen Drogenentzug. Dissertation: Universität Ulm 1999

${ }^{14}$ Murray JB. Psychophysiological Aspects of Amphetamine-Methamphetamine Abuse. The Journal of Psychology 1998; 132: 227-237

15 Darke S, Kaye S, Ross J. Transitions between the injection of heroin and amphetamines. Addiction 1999; 94: 1795-1803

${ }^{16}$ Rawson R, Huber A, Brethen P, Obert J, Gulati V, Shoptaw S, Ling W. Methamphetamine and Cocaine Users: Differences in Characteristics and Treatment Retention. Journal of Psychoactive Drugs 2000; 32: $233-238$

${ }^{17}$ Schiller M, Link M, Härtel R, Forster St, Reymann G, Wolfersdorf M. Zur heutigen Akutversorgungssituation Drogenabhängiger - Ergebnisse einer Umfrage in deutschen Kliniken für Psychiatrie und Psychotherapie 1999 . Suchttherapie $2001 ; 2$ : 235-236 